\title{
Monoclonal antibody targeting complement C9 binding domain of Trichinella spiralis paramyosin impairs the viability of Trichinella infective larvae in the presence of complement
}

\author{
Yuwan Hao, Xi Zhao, Jing Yang, Yuan Gu, Ran Sun and Xinping Zhu*
}

\begin{abstract}
Background: Trichinella spiralis expresses paramyosin (Ts-Pmy) not only as a structural protein but also as an immunomodulator that inhibits host complement as a survival strategy. Previous studies demonstrated that Ts-Pmy bound to complement components C8 and C9 and inhibited the polymerization of C9 during the formation of the membrane attack complex (MAC). The C9 binding domain of Ts-Pmy was identified within 14 amino acid residues at the C-terminus of Ts-Pmy. The production of a monoclonal antibody that specifically targets the C9 binding site is necessary for further studies of Ts-Pmy function and may be used as a therapeutic agent for T. spiralis infection.

Methods: In this study, a monoclonal antibody against the complement C9 binding domain of Ts-Pmy (mAb 9G3) was produced using hybridoma technology. The binding activity of the mAb produced for recombinant or native $T_{s}$-Pmy and the blockade of Ts-Pmy binding to C9 by the mAb were assessed by Western blot analysis. The effect of the $\mathrm{mAb}$ on the viability of $T$. spiralis was observed by co-incubation of T. spiralis with $\mathrm{mAb} 9 \mathrm{G} 3$ in the presence of complement in vitro and by passive transfer of the $\mathrm{mAb}$ into naive mice following $T$. spiralis larval challenge.

Results: mAb 9G3 was successfully produced against the C9 binding domain of Ts-Pmy and bound specifically not only to recombinant Ts-Pmy but also to native Ts-Pmy expressed in different stages of $T$. spiralis, including adult worms, newborn larvae and muscle larvae. The binding of mAb $9 G 3$ to Ts-Pmy efficiently blocked the binding of Ts-Pmy to human complement C9, resulting in a significant increase in the complement-mediated killing of newborn larvae in vitro and reduced infectivity of T. spiralis larvae in mice passively transferred with the mAb.

Conclusions: $\mathrm{mAb} 9 \mathrm{G} 3$ is a specific antibody that binds to the C9 binding domain of Ts-Pmy and interferes with Ts-Pmy's complement-binding activity. Therefore, this mAb is a protective antibody that has potential as a preventive and therapeutic agent for T. spiralis infection.
\end{abstract}

Keywords: Trichinella spiralis, Immune evasion, Paramyosin, Monoclonal antibody

\section{Background}

Trichinella spiralis is a parasitic nematode that infects humans and other mammals around the world [1]. Trichinellosis, caused by the consumption of raw or undercooked meat contaminated with infective muscle larvae, remains an important infectious disease on a global scale [2]. Due to the predominantly zoonotic importance of infection, new regulations for meat inspection and efficient

\footnotetext{
* Correspondence: zhuxping@ccmu.edu.cn

Department of Parasitology, School of Basic Medical Sciences, Capital Medical University, Beijing, China
}

quality control measures have been studied and enacted in recent years [3]. In addition, the identification of potential vaccine candidates, proteins and protective antibodies has been used as an important strategy for the control of T. spiralis infection $[4,5]$.

The host complement system is the first line of defense against pathogenic organisms [6]. Blocking the assembly of complement is a pathogens principal mechanism for escaping from host immune attack [7]. Parasitic nematodes have been suggested to produce compounds capable of inhibiting the assembly and polymerization 
of the membrane complex attack, thus preventing complement-mediated damage [8]. Subsequent studies revealed that $T$. spiralis worms could bind to complement components [8-10], suggesting that $T$. spiralis contains proteins that bind to and potentially inhibit complement activation to protect against host complement attack.

Paramyosin, which serves as an essential muscle protein in invertebrates, forms the core of thick myofilaments, which determine the length and stability of muscles [11]. In addition to being a structural protein, paramyosin has been defined as a potential vaccine candidate against some helminthiases [12-15]. Additional evidence demonstrated that paramyosin played an important role as an immunomodulatory protein in helminth infections $[12,14,16]$. Paramyosin, which acts as a complement inhibitor, is capable of inhibiting complement activation by binding to at least three complement components: $\mathrm{C} 1 \mathrm{q}$ [17], C8, and C9 [18-20]. In our previous study, a full-length cDNA encoding T. spiralis paramyosin (Ts-Pmy) was cloned and partial protection against $T$. spiralis infection was achieved in mice by immunizing with recombinant $T s$-Pmy (rTsPmy) $[16,21]$. In addition, paramyosin expressed on the outer membrane of $T$. spiralis plays an important role in host immunomodulation, specifically by binding to human complement components $\mathrm{C} 8$ and $\mathrm{C} 9$ and inhibiting the formation of the complement membrane attack complex (MAC), thus creating an effective strategy via which the Trichinella parasite can evade host complement attack [20]. Our recent results further identified the exact C9 binding site in $T s$-Pmy, which was narrowed to 14 amino acid residues within the $\mathrm{C}$-terminus of $T s$-Pmy between ${ }^{866} \mathrm{Val}$ and ${ }^{879} \mathrm{Met}$ via fragmental expression and synthesized peptide screening [20]. In the presence of the synthesized C9-binding peptide, human C9 polymerization and human complement-mediated hemolytic activity were inhibited [22].

The immune escape function of paramyosin is an effective survival strategy that allows $T$. spiralis to live within its host. Blocking the complement inhibitory activity of paramyosin could be explored as an alternative strategy for the control of $T$. spiralis infection. Monoclonal antibodies (mAbs) targeting the complement $\mathrm{C} 9$ binding site of $T$. spiralis paramyosin were produced and characterized in this study. The viability of $T$. spiralis newborn larvae (NBL) treated with one of these mAbs (mAb 9G3) was impaired in the presence of human serum, and partial protection against $T$. spiralis larval challenge was achieved in mice passively transferred with the mAb against the Ts-Pmy C9 binding domain.

\section{Methods}

\section{Experimental animals}

All experimental animals were purchased from the Laboratory Animal Services Center of Capital Medical
University (Beijing, China). All experimental procedures were reviewed and approved by the Capital Medical University Animal Care and Use Committee and were consistent with the NIH Guide for the Care and Use of Laboratory Animals.

\section{Parasites and antigen preparation}

T. spiralis (ISS 533) was maintained in female ICR mice. Muscle larvae were recovered from infected mice using the standard pepsin digestion method, as described previously [23]. Adult worms were collected from intestines of mice 5-7 days after experimental infection. NBL were obtained from fertile female adult worms cultured overnight in RPMI 1640 containing antibiotics $(100 \mathrm{U} / \mathrm{ml}$ penicillin and $100 \mathrm{mg} / \mathrm{ml}$ streptomycin) at $37^{\circ} \mathrm{C}$ in the presence of $5 \% \mathrm{CO}_{2}$. Crude somatic extracts of adult worms, muscle larvae (ML) and NBL were prepared by homogenizing the parasites in PBS, $\mathrm{pH}$ 7.4, protein concentrations of the extract supernatants were determined using the BCA assay (Pierce, USA).

\section{Synthesis of Ts-Pmy C9 binding domain peptide}

The C9 binding domain in Ts-Pmy was identified in our previous study [20], and the binding peptide P25 (KHRSSVSMGKSLSSKVYVMEEGHEY, Ts-Pmy861-885), which contains the sequence of the C9 binding site (VSMGKSLSSKVYVM, Ts-Pmy866-879), was synthesized using the solid-phase peptide synthesis method, purified up to $95 \%$ via preparative RP-HPLC and verified by mass spectrometry (Aviva Bio, China).

\section{Production of monoclonal antibodies}

To increase the immunogenicity of peptide P25, BSA was used as a carrier. Monoclonal antibodies (mAbs) against peptide P25 were produced using hybridoma technology [24]. Briefly, female BALB/c mice (6-8 weeks old) were immunized subcutaneously with $100 \mu \mathrm{g}$ of BSA conjugated-P25 emulsified with an equal volume of Freund's complete adjuvant, and boosted twice at 2week intervals with the same amount of BSA-conjugated peptide emulsified with an equal volume of Freund's incomplete adjuvant. Three days after the final boost, the mice were sacrificed and the spleen cells were removed and fused with SP2/0 cells at a ratio of 5:1 with $50 \%$ $(\mathrm{w} / \mathrm{v})$ pre-warmed $\left(37^{\circ} \mathrm{C}\right)$ PEG2000 (Sigma-Aldrich, USA). The hybridoma supernatants were screened for antibody activity via ELISA using synthesized peptide P25 as the antigen. The hybridomas secreting antibodies were cloned by limiting dilution. The hybridoma cell clone 9G3, which exhibited stable growth and secreted a high titer of antibody, was selected for further analysis and for the production of ascites in the peritoneal cavities of BALB/c mice.

The mAb-containing culture supernatant and ascitic fluid were collected from BALB/c mice, and the antibody 
titer was determined using ELISA. The mAbs were purified by affinity chromatography using a Protein A sepharose 4 FF column (GE Healthcare, USA). The subclass of the mAb was determined using a Mouse Monoclonal Antibody Isotyping Kit (Gibco-BRL, USA).

\section{Immunological recognition of $\mathrm{mAb} 9 \mathrm{G3}$}

To determine whether mAb $9 \mathrm{G} 3$ enables the recognition of recombinant or native $T s$-Pmy, recombinant $\mathrm{r} T s$-Pmy was expressed in E. coli BL21 as described previously [20]. Purified rTs-Pmy $(0.5 \mu \mathrm{g})$, native $T s$-Pmy containing crude somatic extracts of ML, NBL or adult worms (5 $\mu \mathrm{g}$ each) and recombinant heat shock protein-70 of T. spiralis (Ts-Hsp70) [25] (1 $\mu \mathrm{g})$, which served as a nonrelevant recombinant protein control, were separated via $12 \%$ SDS-PAGE under reducing conditions, and transferred onto a nitrocellulose (NC) membrane (Millipore, USA). After blocking with 5\% milk in PBS for $1 \mathrm{~h}$ at room temperature, the membrane was incubated with mAb 9G3 $(0.2 \mu \mathrm{g} / \mathrm{ml})$ in $1 \%$ skimmed milk-PBS for $1 \mathrm{~h}$ at room temperature. IRDye $800 \mathrm{CW}$-labeled goat anti-mouse IgG (LI-COR, Germany) was used as the secondary antibody. Recognition was detected and imaged using the Odyssey infrared imaging system (LI-COR, Germany).

To determine whether mAb 9G3 is able to recognize native $T s$-Pmy expressed on the surface of the parasite, longitudinal sections of $T$. spiralis ML were prepared. The sections were blocked with normal goat serum (1:10) for $30 \mathrm{~min}$ and subsequently incubated with $5 \mu \mathrm{g} / \mathrm{ml}$ of $9 \mathrm{G} 3$ in $1 \times$ PBS, pH 7.4 with $0.05 \%$ Tween-20 (PBST) for $1 \mathrm{~h}$ at room temperature. Normal mouse serum was used at a 1:100 dilution as a control. The sections were washed with PBST and subsequently incubated with a 1:200 dilution of an Alexa Fluor 488-labeled goat anti-mouse IgG antibody for $1 \mathrm{~h}$, followed by the addition of the DAPI fluorescent nuclear stain $(1.25 \mu \mathrm{g} / \mathrm{ml})$. The labeling images were obtained via confocal laser scanning microscopy.

\section{Inhibition of $\mathrm{rTs}$-Pmy binding to $\mathrm{C} 9$ by mAb $9 \mathrm{G} 3$}

To determine whether mAb 9G3 against the C9 binding domain of $T s$-Pmy is able to inhibit the binding of rTs-Pmy to human complement C9, rTs-Pmy $(2 \mu \mathrm{g})$ was transferred onto a NC membrane (Millipore, USA) and subsequently incubated with various amounts of mAb 9G3 $(0,2$, and $4 \mu \mathrm{g})$ for $1 \mathrm{~h}$ at room temperature. BSA or $\mathrm{mAb}$ 7E2 (10 $\mu \mathrm{g}$ each), which is another monoclonal antibody against rTs-Pmy1-315 that lacks the ability to bind to the complement binding site [26], were used as controls for the binding assay. After washing, the membrane strips were incubated with human C9 $(1 \mu \mathrm{g} / \mathrm{ml})$ (Merck, Germany) at $37^{\circ} \mathrm{C}$ for $2 \mathrm{~h}$ and subsequently incubated with a rabbit anti-C9 polyclonal antibody $(0.2 \mu \mathrm{g} / \mathrm{ml})$ (Abnova, Taiwan) for $1 \mathrm{~h}$ at room temperature. IRDye $800 \mathrm{CW}$-conjugated goat anti-rabbit IgG (50 ng/ml) (LI-COR, Germany) was used as the secondary antibody. All membrane strips were detected and imaged using the Odyssey infrared imaging system (LI-COR, Germany).

\section{mAb $9 \mathrm{G} 3$ enhanced complement-mediated killing of NBL in vitro}

A previous study demonstrated that $\mathrm{r} T s$-Pmy could bind to complement components C8 and C9 and inhibit the complement-mediated killing of NBL [20]. To determine whether blocking the Ts-Pmy C9-binding domain with mAb 9 G3 would enhance the complement-mediated killing of $T$. spiralis larvae, freshly obtained NBL were pretreated with different amounts of mAb 9G3 (2, 20, or $40 \mu \mathrm{l}$ of a $1 \mathrm{mg} / \mathrm{ml}$ solution) in a final volume of $150 \mu \mathrm{l} /$ well in a 96-well plate for $30 \mathrm{~min}$ at room temperature. The same amount of mAb 7E2 was used as non-relevant antibody control and normal mouse serum was used as negative antibody control. Subsequently, $100 \mu \mathrm{l}$ of fresh normal human serum was added into each well as a source of complement for an overnight incubation at $37^{\circ} \mathrm{C}$ in a $5 \% \mathrm{CO}_{2}$ incubator. Heat-inactivated human serum $\left(30 \mathrm{~min}\right.$ at $56^{\circ} \mathrm{C}$ ) served as a control. The mortality of the NBL after the incubation was assessed based on motility under an inverted microscope (worms without any movement during 30 seconds of observation and total stretchout were scored as dead) [18]. The experiments were performed in triplicate. The percent mortality was calculated in the complement-treated group compared with the heat-inactivated serum group.

\section{Passive immunization and parasite challenge}

To evaluate the possible protective function of $\mathrm{mAb}$ 9G3 in the defense against T. spiralis infection via blocking the C9-binding site of surface-expressed Ts-Pmy, a group of six female BALB/c mice that were six to eight weeks of age underwent passive transfer with $0.5 \mathrm{mg}$ of mAb 9G3 in a total volume of $0.1 \mathrm{ml}$ per mouse $2 \mathrm{~h}$ before oral challenge with $500 \mathrm{~T}$. spiralis ML. Another boost containing the same amount of mAb 9G3 was administered 4 days after infection. T. spiralis-infected sera and normal mouse sera at the same dose were used as the positive and negative controls, respectively. Infected mouse sera were obtained from BALB/c mice 45 days post-infection with $500 \mathrm{~T}$. spiralis ML. The reduction of the worm burden was evaluated by counting the number of muscle larvae collected from whole muscle tissue of mice 45 days after the challenge infection [16].

\section{Statistical analysis}

The data were expressed as the mean \pm standard error (S.E.). Statistical analysis was performed with GraphPad Prism software (San Diego, USA) using One-Way ANOVA. Differences for which $\mathrm{P}<0.05$ were considered statistically significant. 


\section{Results}

Production and characterization of $\mathrm{mAb} 9 \mathrm{G} 3$

Monoclonal antibodies (mAbs) against the Ts-Pmy C9 binding site were made by fusing splenocytes from mice immunized with the C9-binding site peptide (P25) with SP2/0. A total of five positive hybridoma cell lines were cloned by limiting dilution based on their high titers against peptide P25 during ELISA, these cell lines were designated as 5E9, 9G3, $10 \mathrm{~F} 10,14 \mathrm{C} 10$, and $14 \mathrm{H} 9$. The subclasses of all of these mAbs were determined to be IgG1. One of these antibodies, mAb 9G3, exhibited the highest absorbance value during ELISA and also exhibited stable growth. Therefore, this clone was selected for further study. The 9G3 hybridoma cells were injected into BALB/c mice for ascitic fluid production. The antibody titers of the cell culture supernatants and the ascitic fluid against P25 were 1:3 200 and 1:516 000, respectively. Western blot analysis revealed that mAb 9G3 recognized not only the full-length recombinant $\mathrm{r} T s$-Pmy protein $(\sim 110 \mathrm{kDa})$ but also the native protein in adult and larval worms of T. spiralis (Figure 1).

\section{Immunolocalization analysis}

An immunofluorescence assay demonstrated that the anti-Ts-Pmy C9 binding site mAb 9G3 could strongly recognize native $T s$-Pmy expressed on the surface of $\mathrm{ML}$ longitudinal sections (Figure 2). This finding is consistent with our previous study, which demonstrated that $T s$-Pmy was expressed on the outer membrane of the cuticle of NBL and adult worms [20]. No significant staining was observed using normal mouse serum at the same dilution. All larvae exhibited nuclei that stained with DAPI.

\section{mAb 9G3 blocks rTs-Pmy binding to C9}

The binding of $\mathrm{r} T s$-Pmy to C9 was significantly inhibited by mAb $9 \mathrm{G} 3$ in a dose dependent manner. When the amount of mAb $9 \mathrm{G} 3$ was increased to $4 \mu \mathrm{g}$, the antibody nearly completely blocked the binding of rTs-Pmy $(2 \mu \mathrm{g})$ to $\mathrm{C} 9(1 \mu \mathrm{g} / \mathrm{ml})$ (Figure 3$)$. As expected, the negative controls mAb 7E2 and BSA exerted no inhibitory effect on the binding of rTs-Pmy to C9, even at high concentrations $(10 \mu \mathrm{g})$.

\section{Enhanced complement-mediated killing of NBL incubated} with mAb 9G3

A previous study demonstrated that Ts-Pmy plays an important role in protecting Trichinella parasites, particularly NBL, from being attacked by host complement by binding to $\mathrm{C} 8$ or $\mathrm{C} 9$ [18]. In this study, NBL were incubated with mAb $9 \mathrm{G} 3$ to block the binding site of native surface-expressed Ts-Pmy for complement C9. The NBL were xthen incubated with fresh human serum as a source of complement. The results demonstrated that the complement-mediated killing of NBL was significantly enhanced by incubation with mAb 9G3 in a dosedependent manner compared with the normal mouse serum group. When up to $40 \mu \mathrm{l}$ of a $1 \mathrm{mg} / \mathrm{ml}$ solution of mAb $9 \mathrm{G} 3$ was added, $32.9 \%$ of NBL were killed compared with $4.3 \%$ of NBL in the normal mouse sera control group ( $40 \mu \mathrm{l}$ added). No significant increases in larval mortality were observed in the group incubated with the monoclonal antibody mAb 7E2 until the amount of mAb 7E2 reached $40 \mu \mathrm{l}$ of a $1 \mathrm{mg} / \mathrm{ml}$ antibody solution, however, the mortality $(10.8 \%)$ of this treatment was much lower than that caused by the same amount of mAb 9G3 (32.9\%) (Figure 4).

\section{Protection against challenge infection in mice adoptively transferred with $\mathrm{mAb} 9 \mathrm{G} 3$}

The challenge results demonstrated that after the passive transfer of $0.5 \mathrm{mg}$ of mAb 9G3 in a total volume of $100 \mu \mathrm{l}$ to $\mathrm{BALB} / \mathrm{c}$ mice, each mouse exhibited a $42.6 \%$ reduction in muscle larvae burden compared with the normal mouse sera transfer control $(P<0.01)$ (Figure 5$)$. As a positive control, mice that received the same volume of $T$. spiralisinfected mouse sera $(100 \mu \mathrm{l})$ displayed a $34.3 \%$ reduction in muscle larvae burden $(P<0.05)$. The results presented here demonstrate that mAb $9 \mathrm{G} 3$ provides protective

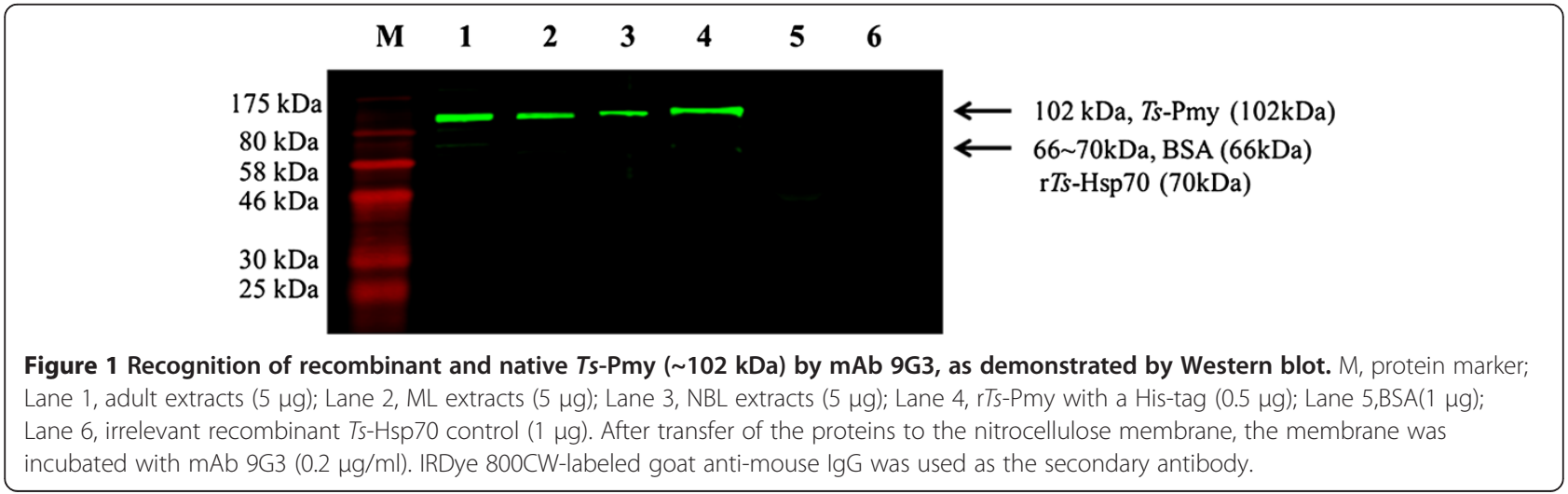




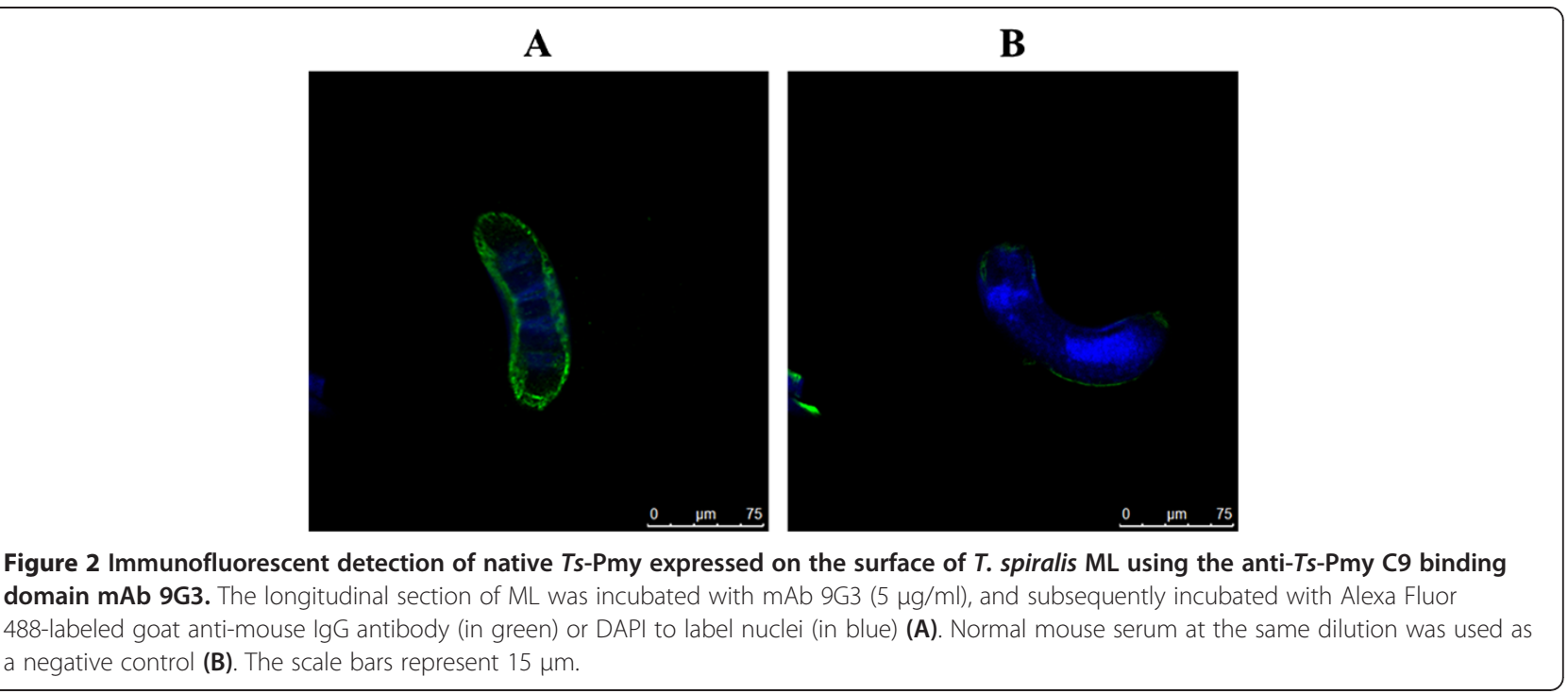

immunity against $T$. spiralis infection that is similar to or better than that induced by $T$. spiralis-infected sera.

\section{Discussion}

Due to its ability to rapidly recognize and eliminate microorganisms, the complement system is an essential and efficient component of the human innate immune system [6]. During co-evolution with their hosts, parasites developed a multitude of mechanisms for evading attacks by the host immune system. Parasite-produced compounds that bind to and inactivate the host's complement components have been identified in several parasites as effective immune escape mechanisms [4,6-8,16,17]. Paramyosin is one of the most important molecules produced by helminths to defense from the host's complement attack $[10,12,14]$. In particular, $T$. spiralis-produced paramyosin (Ts-Pmy) was demonstrated to bind to human complement components C8 and C9 and subsequently inhibit the polymerization of $\mathrm{C} 9$ during the formation of the membrane attack complex (MAC), thus protecting Trichinella larvae from being attacked by the host complement system [20]. The complement C9 binding domain of Ts-Pmy was identified within 14 amino acid residues $\left({ }^{866} \mathrm{Val}^{879} \mathrm{Met}\right)$ at the C-terminus of Ts-Pmy [22]. Theoretically, a specific antibody targeting the complement binding domain of Ts-Pmy should block the binding of $T s$-Pmy to human complement, exposing the parasites to attack by the host complement system. Therefore, such an antibody would be a protective antibody against T.spiralis infection.

To produce specific antibodies targeting the C9 binding domain of Ts-Pmy, a 25 amino-acid peptide (P25) covering Ts-Pmy861-885, including the 14 amino-acid sequence of the C9 binding site (VSMGKSLSSKVYVM, Ts-Pmy866-879), was synthesized and used to immunize mice for the production of monoclonal antibodies. Although the C9 binding site in Ts-Pmy was narrowed to 14 amino acid residues $\left({ }^{866} \mathrm{Val}_{-}{ }^{879} \mathrm{Met}\right)$, the extension of the amino acid sequence on both sides up to 25 amino acids may increase the immunogenicity of the peptide. The production of monoclonal antibodies against synthetic peptides is a versatile tool in the molecular and functional analysis of proteins [27-29]. However, some factors should be considered during the design of peptides for the production of anti-peptide antibodies, including the length of the peptides, the location of the

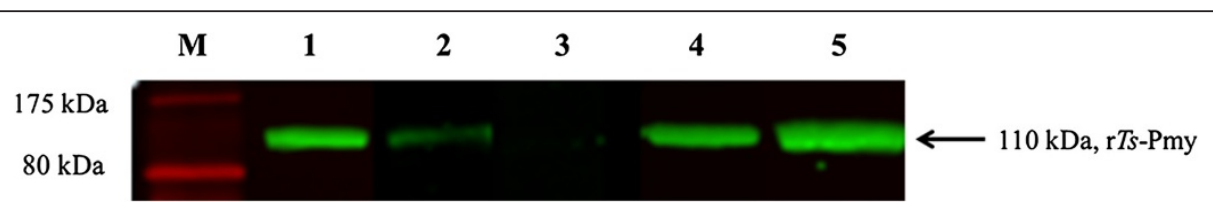

Figure 3 Inhibition of rTs-Pmy binding to C9 by the addition of different amounts of mAb 9G3, as determined by Western blot. rTs-Pmy $(2 \mu \mathrm{g})$ was transferred onto a NC membrane and incubated with various amounts of mAb 9G3. Lane 1: PBS only; Lane 2: mAb 9G3 (2 $\mu$ g); Lane 3: mAb $9 \mathrm{G} 3(4 \mu \mathrm{g})$. Lane 4: BSA $(10 \mu \mathrm{g})$ and Lane 5: irrelevant mAb 7E2 control $(10 \mu \mathrm{g})$. After washing, the membrane was incubated with human C9 $(1 \mu \mathrm{g} / \mathrm{ml})$ at $37^{\circ} \mathrm{C}$ for $2 \mathrm{~h}$ and subsequently incubated with rabbit anti-C9 polyclonal antibody $(0.2 \mu \mathrm{g} / \mathrm{ml})$ for $1 \mathrm{~h}$ at room temperature. IRDye $800 \mathrm{CW}$-labeled goat anti-rabbit lgG $(50 \mathrm{ng} / \mathrm{ml})$ was used as the secondary antibody. 


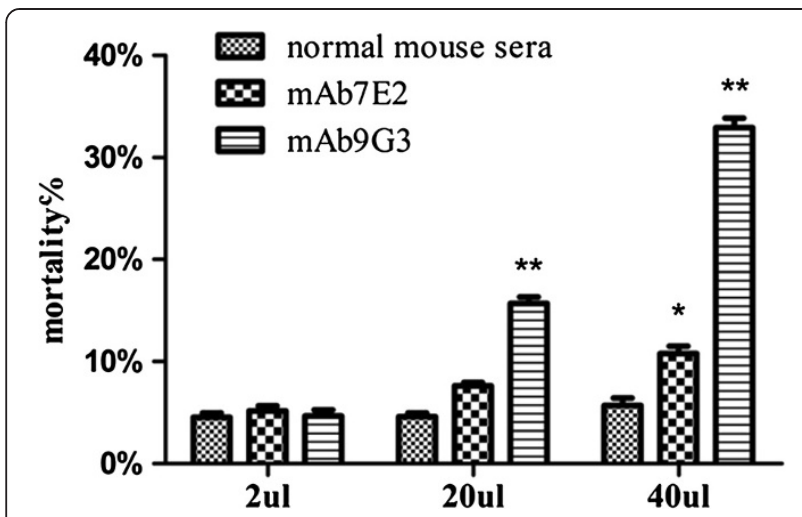

Figure 4 Enhanced complement-mediated killing of $T$. spiralis NBL incubated with $\mathrm{mAb} 9 \mathrm{G} 3$. NBL were pretreated with different volumes $(2 \mu \mathrm{l}, 20 \mu \mathrm{l}$ and $40 \mu \mathrm{l})$ of $\mathrm{mAb} 9 \mathrm{G} 3(1 \mathrm{mg} / \mathrm{ml})$, the irrelevant $\mathrm{mAb} 7 \mathrm{E} 2(1 \mathrm{mg} / \mathrm{ml})$ or normal mouse serum prior to the addition of $100 \mu \mathrm{l}$ of normal human serum. Heat-inactivated human serum served as a control. The mortality of the NBL was assessed under an inverted microscope. The results are presented as the arithmetic mean \pm standard error (SE) from triplicate experiments. ${ }^{* *} p<0.01$, ${ }^{*} p<0.05$ compared with the normal mouse sera control.

peptides in the native molecule and hydrophilicity of the peptides [30]. In this study, the synthesized P25 peptide was conjugated to BSA to increase its immunogenicity. To exclude the possible production of mAbs against the conjugated BSA, the hybridoma culture supernatants

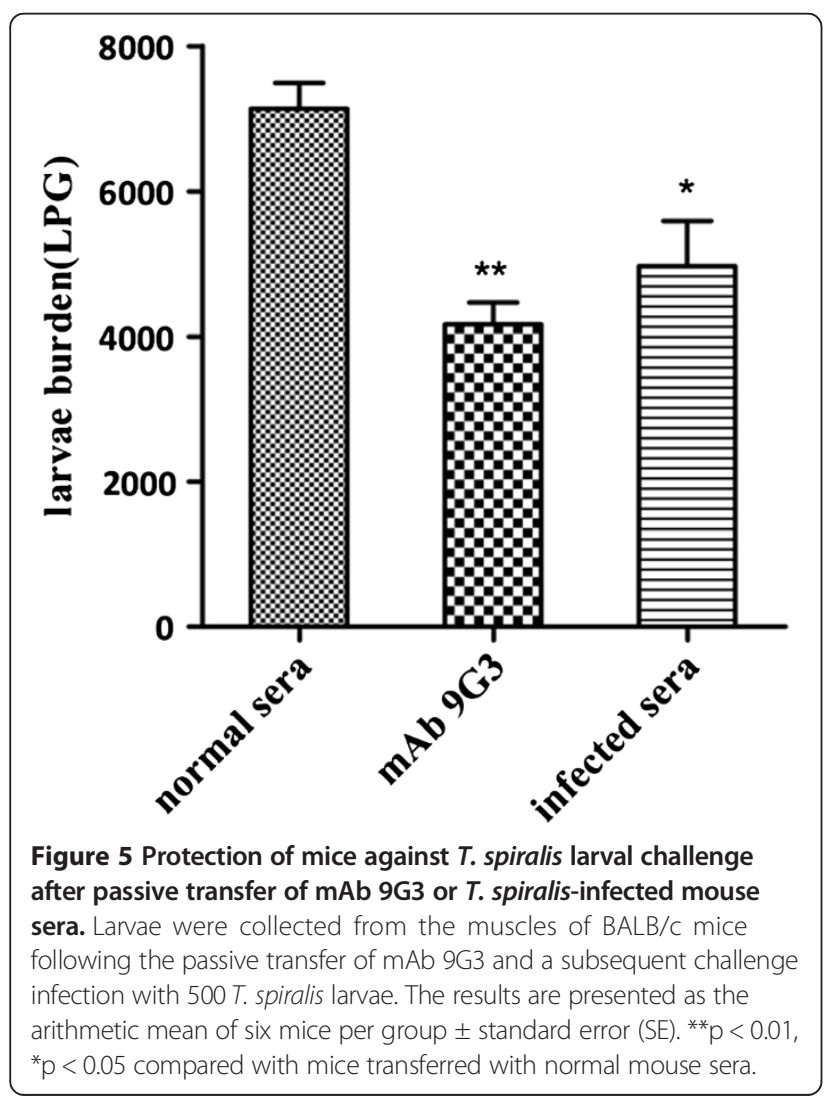

were screened directly against synthesized peptide as an antigen coated on plate during ELISA. Among the five stable hybridoma cell lines, only the stable cell line 9G3 secreted an antibody that recognized not only full-length recombinant $T s$-Pmy, but also native $T s$-Pmy in $T$. spiralis adult and larval (ML and NBL) extracts. Immunolocalization on longitudinal sections of ML demonstrated that mAb 9 G3 also recognized Ts-Pmy expressed on the surface of Trichinella larva, which is consistent with our previous study that demonstrated that Ts-Pmy was present on the outer membrane of the cuticle of adults and NBL [18]. The universal distribution of Ts-Pmy in different developmental stages of $T$. spiralis provides a more adequate experimental basis for the role of paramyosin as a potential modulator of the host immune system during different parasitic stages.

In addition to the specific binding of mAb 9G3 to native $T s$-Pmy expressed in different developmental stages of $T$. spiralis, this antibody also efficiently inhibits the binding of Ts-Pmy to human complement C9, suggesting that this antibody possesses the ability to functionally block the complement binding activity of Ts-Pmy. To determine whether blocking the C9-binding domain of $T s$-Pmy would inhibit the protection employed by $T s$ Pmy against complement attack and therefore enhance the complement-mediated killing of T. spiralis parasites, we incubated $T$. spiralis NBL with mAb 9 G3 before adding fresh human serum as a source of complement. NBL were chosen as a target for the complement killing assay because the NBL stage is the stage that migrates through the blood and lymphatic circulation to muscular tissue and is exposed directly to the host immune system, thus, this stage should develop sophisticated abilities to evade complement attack, such as complement-binding Ts-Pmy [18], as a survival strategy [5]. Blocking Ts-Pmy on the surface of $T$. spiralis NBL by incubating live NBL with mAb 9G3 resulted in significantly enhanced complementmediated killing of NBL in the presence of fresh human serum in vitro, suggesting that blocking the C9-binding domain of Ts-Pmy with mAb 9G3 efficiently inhibits the function of Ts-Pmy that protects the parasite from being attacked by host complement. After incubation with $\mathrm{mAb}$ 7E2, which is a mAb that binds to the $\mathrm{N}$-terminus of $T s$-Pmy but not to the complement-binding domain of $T s$-Pmy, T. spiralis larvae exhibited similar levels of viability or reduced levels of viability when large amounts of antibody were added, however, the complement-mediated killing of larvae was much lower than that induced by incubation with mAb 9G3 (Figure 4). The limited complement-mediated larval killing by a $\mathrm{mAb}$ that does not bind to the complement-binding domain of Ts-Pmy (mAb 7E2) may be a result of complement activation through the classic antibody-mediated pathway. The significantly higher complement-mediated larval killing by 
the anti-C9 binding domain of $T s$-Pmy mAb than by the $\mathrm{mAb}$ that is not related to the complement binding site of $T s$-Pmy further indicates that Ts-Pmy protects Trichinella parasites from being attacked by host complement by inhibiting the alternative pathway of complement activation [6]. Impaired infectivity of $T$. spiralis parasites was also observed in mice passively transferred with the anti-C9 binding domain of Ts-Pmy mAb 9G3. The protection level induced by mAb $9 \mathrm{G} 3(42.6 \%)$ was higher than that elicited by $T$. spiralis infected mouse sera (34.3\%) (Figure 5 ).

This study further elucidates the mechanism of nematode paramyosin involved in the immunomodulation of host immune response and the specific monoclonal antibody against paramyosin's functional domain could be used as a therapeutic or preventive agent for Trichinellosis. However, except for the binding site for $\mathrm{C} 9$ on Trichinella-secreted paramyosin, there may be more binding sites for other components of complement that may also contribute to evading complement attack for the survival of parasite in the host, which is under investigation.

\section{Conclusions}

In summary, in this study, we demonstrated that the monoclonal antibody 9G3 that targets the complement C9 binding domain of Ts-Pmy could efficiently block the complement inhibitory activity of Ts-Pmy, resulting in a significant increase in the complement-mediated killing of newborn larvae in vitro and reduced infectivity of T. spiralis larvae in mice passively transferred with the $\mathrm{mAb}$. Therefore, mAb $9 \mathrm{G} 3$ is a protective antibody that has potential as a preventive and therapeutic agent for T. spiralis infection.

\section{Competing interests}

The authors declare that they have no competing interests.

\section{Authors' contributions}

YWH performed the experiments and drafted the manuscript. $X Z, J Y, Y G$, and RS performed some of the experiments. XPZ designed the study and revised the manuscript. All authors read and approved the final manuscript.

\section{Acknowledgements}

This study was supported by grants from the National Natural Science Foundation of China $(81171598,81371837,81201313)$, the National Science and Technology Major Project (2012ZX10004220-012), and Collaborative Innovation Center of Infectious Diseases. We thank Zhifei Zhang, Jingjing Huang, Fengyun Wang and Jin Pan for their technical assistance.

Received: 27 May 2014 Accepted: 1 July 2014

Published: 4 July 2014

\section{References}

1. Gottstein B, Pozio E, Nockler K: Epidemiology, diagnosis, treatment, and control of trichinellosis. Clin Microbiol Rev 2009, 22(1):127-145.

2. Dupouy-Camet J: Trichinellosis: a worldwide zoonosis. Vet Parasitol 2000, 93(3-4):191-200.

3. Dupouy-Camet J: Presidential address of ICT12 Conference: "Trichinella and trichinellosis-a never ending story". Vet Parasitol 2009, 159(3-4):194-196.

4. Zhang YL, Wang ZQ, Li LG, Cui J: Molecular characterization of Trichinella spiralis aminopeptidase and its potential as a novel vaccine candidate antigen against trichinellosis in BALB/c mice. Parasit Vectors 2013, 6(1):246.
5. Zumaquero-Rios JL, Garcia-Juarez J, De-la-Rosa-Arana JL, Marcet R, Sarracent-Perez J: Trichinella spiralis: monoclonal antibody against the muscular larvae for the detection of circulating and fecal antigens in experimentally infected rats. Exp Parasitol 2012, 132(4):444-449.

6. Lambris JD, Ricklin D, Geisbrecht BV: Complement evasion by human pathogens. Nat Rev Microbiol 2008, 6(2):132-142.

7. Bruschi F: The immune response to the parasitic nematode Trichinella and the ways to escape it. From experimental studies to implications for human infection. Curr Drug Targets Immune Endocr Metabol Disord 2002, 2(3):269-280.

8. Hong Y, Kim CW, Ghebrehiwet B: Trichinella spiralis: activation of complement by infective larvae, adults, and newborn larvae. Exp Parasitol 1992, 74(3):290-299.

9. Kennedy MW, Kuo YM: The surfaces of the parasitic nematodes Trichinella spiralis and Toxocara canis differ in the binding of post-C3 components of human complement by the alternative pathway. Parasite Immunol 1988, 10(4):459-463.

10. Nareaho A, Saari S, Meri S, Sukura A: Complement membrane attack complex formation and infectivity of Trichinella spiralis and T. nativa in rats. Vet Parasitol 2009, 159(3-4):263-267.

11. Elfvin M, Levine RJ, Dewey MM: Paramyosin in invertebrate muscles. I. Identification and localization. J Cell Biol 1976, 71(1):261-272.

12. Lanar DE, Pearce EJ, James SL, Sher A: Identification of paramyosin as schistosome antigen recognized by intradermally vaccinated mice. Science 1986, 234(4776):593-596.

13. Nanduri J, Kazura JW: Paramyosin-enhanced clearance of Brugia malayi microfilaremia in mice. J Immunol 1989, 143(10):3359-3363.

14. Muhlschlegel F, Sygulla L, Frosch P, Massetti P, Frosch M: Paramyosin of Echinococcus granulosus: cDNA sequence and characterization of a tegumental antigen. Parasitol Res 1993, 79(8):660-666.

15. Ferrer E, Moyano E, Benitez L, Gonzalez LM, Bryce D, Foster-Cuevas M, Davila I, Cortez MM, Harrison LJ, Parkhouse RM, Garate T: Cloning and characterization of Taenia saginata paramyosin cDNA. Parasitol Res 2003, 91(1):60-67.

16. Yang J, Yang Y, Gu Y, Li Q, Wei J, Wang S, Boireau P, Zhu X: Identification and characterization of a full-length CDNA encoding paramyosin of Trichinella spiralis. Biochem Biophys Res Commun 2008, 365(3):528-533.

17. Laclette JP, Shoemaker CB, Richter D, Arcos L, Pante N, Cohen C, Bing D, Nicholson-Weller A: Paramyosin inhibits complement C1. J Immunol 1992, 148(1):124-128.

18. Deng J, Gold D, LoVerde PT, Fishelson Z: Mapping of the complement C9 binding domain in paramyosin of the blood fluke Schistosoma mansoni. Int J Parasitol 2007, 37(1):67-75

19. Deng J, Gold D, LoVerde PT, Fishelson Z: Inhibition of the complement membrane attack complex by Schistosoma mansoni paramyosin. Infect Immun 2003, 71(11):6402-6410.

20. Zhang Z, Yang J, Wei J, Yang Y, Chen X, Zhao X, Gu Y, Cui S, Zhu X: Trichinella spiralis paramyosin binds to $C 8$ and $C 9$ and protects the tissue-dwelling nematode from being attacked by host complement. PLoS Negl Trop Dis 2011, 5(7):e1225.

21. Yang J, Gu Y, Yang Y, Wei J, Wang S, Cui S, Pan J, Li Q, Zhu X: Trichinella spiralis: immune response and protective immunity elicited by recombinant paramyosin formulated with different adjuvants. Exp Parasitol 2010, 124(4):403-408.

22. Zhao X, Hao Y, Yang J, Gu Y, Zhu X: Mapping of the complement C9 binding domain on Trichinella spiralis paramyosin. Parasit Vectors 2014 7(1):80.

23. Dennis DT, Despommier DD, Davis N: Infectivity of the newborn larva of Trichinella spiralis in the rat. J Parasitol 1970, 56(5):974-977.

24. Yokoyama WM: Production of Monoclonal Antibodies. In Current Protocols in Immunology. 1995:2-5.

25. Wang S, Zhu X, Yang Y, Yang J, Gu Y, Wei J, Hao R, Boireau P, Cui S: Molecular cloning and characterization of heat shock protein 70 from Trichinella spiralis. Acta Trop 2009, 110(1):46-51.

26. Wei J, Gu Y, Yang J, Yang Y, Wang S, Cui S, Zhu X: Identification and characterization of protective epitope of Trichinella spiralis paramyosin. Vaccine 2011, 29(17):3162-3168.

27. lankov ID, Penheiter AR, Carlson SK, Galanis E: Development of monoclonal antibody-based immunoassays for detection of Helicobacter pylori neutrophil-activating protein. J Immunol Methods 2012, 384(1-2):1-9. 
28. Maleki LA, Majidi J, Baradaran B, Abdolalizadeh J, Akbari AM: Production and characterization of murine monoclonal antibody against synthetic peptide of CD34. Hum Antibodies 2013, 22(1):1-8.

29. Kajiura S, Yashiki T, Funaoka H, Ohkaru Y, Nishikura K, Kanda T, Ajioka Y, Igarashi M, Hatakeyama K, Fujii H: Establishment and characterization of monoclonal and polyclonal antibodies against human intestinal fatty acid-binding protein (I-FABP) using synthetic regional peptides and recombinant I-FABP. J Immunoassay Immunochem 2008, 29(1):19-41.

30. Tanaka T, Slamon DJ, Cline MJ: Efficient generation of antibodies to oncoproteins by using synthetic peptide antigens. Proc Natl Acad Sci U S A 1985, 82(10):3400-3404.

doi:10.1186/1756-3305-7-313

Cite this article as: Hao et al: Monoclonal antibody targeting complement C9 binding domain of Trichinella spiralis paramyosin impairs the viability of Trichinella infective larvae in the presence of complement. Parasites \& Vectors 2014 7:313.

\section{Submit your next manuscript to BioMed Central and take full advantage of:}

- Convenient online submission

- Thorough peer review

- No space constraints or color figure charges

- Immediate publication on acceptance

- Inclusion in PubMed, CAS, Scopus and Google Scholar

- Research which is freely available for redistribution 